\title{
A Stellar-mass Black Hole in the Ultra-luminous X-ray Source M82 X-1
}

\author{
Takashi Okajima ${ }^{1}$ \\ Code 662, Exploration of the Universe Division, NASA's Goddard Space Flight Center, \\ Greenbelt, $M D$ 20771 \\ Ken Ebisawa \\ Institute of Space and Astronautical Science, 3-1-1 Yoshinodai, Sagamihara, Kanagawa, \\ 229-8510, Japan \\ and \\ Toshihiro Kawaguchi \\ Department of Physics and Mathematics, Aoyama Gakuin University, Fuchinobe 5-10-1, \\ Sagamihara, Kanagawa 229-8558, Japan
}

\begin{abstract}
We have analyzed the archival XMM-Newton data of the archetypal UltraLuminous X-ray Source (ULX) M82 X-1 with an $105 \mathrm{ksec}$ exposure when the source was in the steady state. Thanks to the high photon statistics from the large effective area and long exposure, we were able to discriminate different $\mathrm{X}$-ray continuum spectral models. Neither the standard accretion disk model (where the radial dependency of the disk effective temperature is $T(r) \propto r^{-3 / 4}$ ) nor a power-law model gives a satisfactory fit. In fact, observed curvature of the M82 X-1 spectrum was just between those of the two models. When the exponent of the radial dependence $\left(p\right.$ in $\left.T(r) \propto r^{-p}\right)$ of the disk temperature is allowed to be free, we obtained $p=0.61_{-0.02}^{+0.03}$. Such a reduction of $p$ from the standard value $3 / 4$ under extremely high mass accretion rates is predicted from the accretion disk theory as a consequence of the radial energy advection. Thus, the accretion disk in M82 X-1 is considered to be in the Slim disk state, where an
\end{abstract}

\footnotetext{
${ }^{1}$ Department of Physics and Astronomy, The Johns Hopkins University
} 
optically thick Advection Dominant Accretion Flow (ADAF) is taking place. We have applied a theoretical slim disk spectral model to M82 X-1, and estimated the black hole mass $\approx 19-32 M_{\odot}$. We conclude that M $82 \mathrm{X}-1$ is a stellar black hole which has been produced through evolution of an extremely massive star, shining at a several times the super-Eddington luminosity.

Subject headings: accretion, accretion disks - black hole physics - X-rays: individual (M82 X-1)

\section{Introduction}

Ultra-luminous X-ray Sources (ULXs) in nearby galaxies have typical X-ray luminosities from $10^{39}$ to $10^{41} \mathrm{erg} \mathrm{s}^{-1}$ (e.g., Makishima et al. 2000; Ptak \& Colbert et al. 2004). M82 $\mathrm{X}-1$ is an archetypal ULX which is located off the nucleus of the galaxy and has exhibited $\mathrm{X}$-ray flares as bright as $\sim 10^{41} \mathrm{erg} \mathrm{s}^{-1}$ (Matsumoto \& Tsuru 1999; Matsumoto et al. 2001; Kaaret et al. 2001). If one assumes that its luminosity is less than the Eddington luminosity ( $L_{\mathrm{Edd}}$ ), the mass of the central object must be at least $\sim 700 M_{\odot}$. Hence, M $82 \mathrm{X}-1$ has been considered an intermediate mass black hole candidate (Matsumoto et al. 1999; Kaaret et al. 2001).

Most ULXs; however, including M82 X-1, exhibit X-ray energy spectra which are much harder than what is expected from standard accretion disks around intermediate black holes (Makishima et al. 2000). In fact, the characteristic color temperature of the standard disk shining at the Eddington luminosity is $\approx 1 \mathrm{keV}\left(M / 10 M_{\odot}\right)^{-1 / 4}$, which has been confirmed through observations of many galactic black hole candidates. As the black hole mass increases, the disk temperature is expected to decrease; contrarily, ULXs tend to have significantly higher disk temperatures than what is expected from their mass (e.g., Okada et al. 1998; Makishima et al. 2000).

There are two major models to explain the "too hot a disk" problem of ULXs. The first model assumes that the accretion disk is not in the standard disk state where the gravitational energy released is converted into optically thick radiation, but in a slim disk state where radial energy advection is dominant (Watarai, Mizuno \& Mineshige 2001; Mizuno, Kubota $\&$ Makishima 2001; Ebisawa et al. 2003). The competing model assumes that the ULX disks have low temperature $(\lesssim 1 \mathrm{keV})$ as expected for intermediate mass black holes. Such disks are assumed to be shrouded by hot, Compton thick clouds, and the observed X-ray spectra above $\sim 1 \mathrm{keV}$ are due to inverse Compton process (approximated by a power-law) of the seed photons from the low temperature disk (e.g., Miller et al. 2003; Miller, Fabian \& Miller 
2004; Wang et al. 2004).

It is very difficult to distinguish the two competing ULX spectral models, since there have not been high quality $\mathrm{X}$-ray data to reveal small differences in the two models. In this paper, we present a precise spectral analysis of XMM-Newton data of M82 X-1 from a 105 ksec exposure. Thanks to the much better statistics than previous observations, we were able to tightly constrain the spectral models. We show that the slim disk model is preferred to the low temperature disk model for M82 X-1. By applying a theoretical slim disk model to the observed spectrum, we estimate the black hole mass varies from $19 M_{\odot}$ to $37 M_{\odot}$, depending on different assumptions. We conclude that the black hole in M82 X-1 is moderately massive, but still "stellar" in the sense that it is produced at the end of ordinary stellar evolution. Models based on "intermediate mass" black holes are not required to explain the M82 X-1 $\mathrm{X}$-ray energy spectrum.

\section{Observation and Data Analysis}

There are three archival XMM-Newton data sets of M82. Two observations were made on May 6, 2001; for $10 \mathrm{ksec}(\mathrm{ObsID}=0112290401)$ and $29 \mathrm{ksec}(\mathrm{ObsID}=0112290201)$, respectively. The other was made on April 21, 2004 for $105 \mathrm{ksec}$ (ObsID=0206080101), which we analyze in the present paper. The observation was carried out employing the European Photon Imaging Camera (EPIC) PN and MOS in the full window and medium filter mode. Data screening, region selection and event extraction were performed with the standard software package XMM-SAS v 6.1.0. In order to eliminate possible contamination from solar flares, events were selected only when the total off-source count rate is less than 0.17 (MOS) and $0.55(\mathrm{PN})$ counts $\mathrm{s}^{-1}$ in $10-15 \mathrm{keV}$. This leaves 63 (MOS1), 65 (MOS2) and 50 (PN) ksec of useful time with an average count rate of $0.7(\mathrm{MOS})$ and $2.2(\mathrm{PN})$ counts $^{-1}$. In this paper, we will primarily analyze the PN data, which have better statistics. The MOS data gives the same results with slightly larger statistical errors.

We extracted the spectrum of $\mathrm{M} 82 \mathrm{X}-1$ within a radius of $18^{\prime \prime}$ around the point source; this procedure is the same as described in Fiorito et al. (2004) and Strohmayer et al. (2003). $X M M-N e w t o n ' s$ moderate spatial resolution $\left(13^{\prime \prime}-15^{\prime \prime}\right.$ in half power diameter) does not allow us to fully resolve the surrounding faint sources resolved by Chandra, including sources 4, 5 , and 6 in Matsumoto et al. (2001). However, sources 4 and 6 are always at least a factor of 10 fainter than M82 X-1, and source 5 was a factor of 3.4 fainter when M82 X-1 was the faintest (Strohmayer et al. 2003). Thus, contamination from these point sources to our M82 $\mathrm{X}-1$ spectral analysis is insignificant. Chandra also revealed diffuse emission in the central region of M82, which is obvious in the EPIC images and spectra as well. Although we cannot 
eliminate the diffuse thermal component from the M82 X-1 spectra, its flux is less than 10 $\%$ of the point source flux at $E>3 \mathrm{keV}$. Therefore, in order to concentrate on the point source spectral analysis, we limit our spectral fitting to the energy range $E>3 \mathrm{keV}$.

The background spectrum was extracted from a ring of the $2^{\prime}$ outer radius and the $18^{\prime \prime}$ inner radius (within which the M82 X-1 spectrum was extracted), and it was subtracted from the source spectrum after being normalized to the detector area. In this manner, the influence of the detector background and the diffuse background surrounding M82 X-1 is minimized. We also examined a blank sky background taken $6.8^{\prime}$ away from M82 X-1, which is free of the M82 diffuse emission. We have carried out spectral fitting using both background spectra, and confirmed that the difference between two backgrounds does not affect our spectral analysis at $E>3 \mathrm{keV}$.

The pulse-height spectral data were binned by 32 channels, which correspond to twice the energy resolution (FWHM). Fittings were performed from 3 to $11 \mathrm{keV}$. First, we employed a power-law model (to model inverse Compton emission of the soft disk photons; see Section 1) and found that there is a weak iron emission line near $6 \mathrm{keV}$ which may be modeled by a single Gaussian. We did not include the interstellar absorption in the fitting, because neither Galactic nor local absorption (e.g., Tsuru et al. 1997) is significant above $3 \mathrm{keV}$. The photon index is found to be 1.73. The Gaussian line is centered at $6.61 \mathrm{keV}$, and has the equivalent width $87 \mathrm{eV}$. We find $\chi^{2}$ to be 96 with 43 d.o.f. Next, we applied the disk blackbody model (Mitsuda et al. 1984) to approximate a standard accretion disk (Shakura \& Sunyaev 1973), including a Gaussian line with similar parameters to those above. The disk blackbody temperature is found to be $2.77 \pm 0.07 \mathrm{keV}$ ( $90 \%$ confidence level hereafter), and $\chi^{2}=81$ ( 43 d.o.f.). Other fitting parameters are shown in Table 3.

Both the power-low model and the disk blackbody model are rejected with a confidence level of $99.98 \%$. Importantly, if we compare the two model fits, we notice opposite trends in the residual (Fig. 1). Namely, the observed spectrum is slightly "curved" downward while the power-law does not. Also, the observed curvature is not as large as that of the disk blackbody model. This indicates that the observed spectral curvature is just between that of the power-law model and the disk blackbody model.

Therefore, we then attempted the "p-free" disk model (Mineshige et al. 1994; Hirano et al. 1995; Kubota and Makishima 2004; Kubota et al. 2006), where the temperature profile of the accretion disk is given by

$$
T(r)=T_{i n}\left(\frac{r}{r_{i n}}\right)^{-p}
$$

with $r_{i n}, T_{i n}$, and $p$ being free parameters. The disk blackbody model has $p=0.75$, and a smaller $p$ value reduces the spectral curvature and make the spectral shape closer to the 
power-law. We found the best fit parameters $p=0.61_{-0.02}^{+0.03}, T_{i n}=3.73_{-0.40}^{+0.58} \mathrm{keV}$ with $\chi^{2}=55$ (42 d.o.f.). The Gaussian parameters are almost the same as those of the other two models. Statistically, the $p$-free disk model describes the spectral shape best among the three models. We can calculate the $F$-value as a measure of the improvement of $p$-free model relative to the disk blackbody model. We find $F(1,42)=\Delta \chi^{2} / \chi_{\nu}^{2}=(81-55) /(55 / 42)=19.9$. Thus, the improvement of the $p$-free model over the disk blackbody model is significant to a 99.99 $\%$ confidence level.

When the disk luminosity is as high as the Eddington luminosity, an optically-thick Advection Dominated Accretion Flow (ADAF) appears (Abramowicz et al. 1988). Such a flow, often called a slim disk, has very low radiation production efficiency due to photon trapping (Begelman 1978). The low energy spectrum of the slim disk has the form $L_{E} \propto E^{-1}$ (Fukue 2000), while that of the standard disk is $L_{E} \propto E^{0.33}$. Since the disk spectral shape is related to the radial exponent $p$ as $L_{E} \propto E^{3-2 / p}$, the spectral change from the standard disk to the slim disk is equivalent to the reduction of $p$ from 0.75 to 0.5 (Watarai et al. 2000).

Our result of $p=0.61_{-0.02}^{+0.03}$ strongly suggests that energy advection is actually taking place, and that the M82 X-1 disk is not in a standard state, but in a slim disk state. In this paper, we employ our own slim disk model (Kawaguchi 2003) to study M82 X-1 energy spectrum. Kawaguchi (2003) has calculated slim disk spectra under four different assumptions. In Model 1, the local emission is assumed to be modified blackbody, and in Model 2, Comptonization is taken into account. Gravitational redshift is included in Model 3 ; and in Model 4, which is our "best" model, transverse Doppler effects are additionally considered. In Figure 2, we compare the simulated Model 4 spectrum with power-law, disk blackbody, and $p$-free disk model. It is obvious that the simulated Model 4 spectral shape is well-represented by the $p$-free model, and its curvature is just between those of the power-law and disk blackbody. Comparing Figure 1 and 2, we can see that M82 X-1 and the simulated Model 4 share similar spectral characteristics.

Next, we directly fit the M82 X-1 spectrum with our slim disk model. We try all four models with assumptions as listed in Table 1 . We tried models with the viscous parameter $\alpha=0.001,0.01,0.1$ and 1 , and found that $\alpha=1$ always gives the best-fit. We also fit allowing $\alpha$ to be free, with little improvement of the fit. Thus, we show only the results with $\alpha=1$ in Table 1. Fixing the distance to M82 at $2.7 \mathrm{Mpc}$ (e.g., Rieke et al. 1980), there are then only two free parameters, $M$ and $\dot{M}$. Kawaguchi's model calculates the face-on disk flux, and we assume face-on geometry in the following.

As summarized in table 1 , we obtain $M=(19-32) M_{\odot}, \dot{M}=(320-560) \times L_{\text {Edd }} / c^{2}$ depending on the physical processes assumed. In the case of the standard optically thick accretion disk where the inner disk radius is three times the Schwartschild radius, $\dot{M}=$ 
17.5 $L_{\mathrm{Edd}} / c^{2}$ which gives the Eddington luminosity, which suggests that M82 X-1 has extremely high mass accretion rates. However, since slim disks are radiation inefficient, the disk luminosity is not so large as being proportional to the mass accretion rates. Bolometric face-on flux $f_{b o l}$ is obtained as $\sim 3 \times 10^{-11} \mathrm{erg} \mathrm{s}^{-1} \mathrm{~cm}^{-2}$ by numerically integrating the best-fit model spectra over the energy, which is weakly depended on the assumptions. The bolometric disk luminosity is $L_{b o l}=2 \pi d^{2} f_{b o l}$ where $d$ is the distance $(2.8 \mathrm{Mpc})$, so $L_{b o l} \approx 1.4 \times 10^{40} \mathrm{erg} \mathrm{s}^{-1}$. Hence, depending on the assumptions, our slim disk model fits suggest that M82 X-1 is shining at 4 to 6 times the super-Eddington luminosity.

Our slim disk model gives $p=0.54$ (Figure 2), while the observation requires $p=0.61$. This suggests that energy advection in the M82 X-1 accretion disk is not so dominant as our slim disk model predicts. Although there is such a room for future improvements in our model, the current slim disk model fits the data quite well, within $\sim 10 \%$, over the entire energy range of the fitting. Therefore, we believe that our primary results, that the black hole mass is $\approx(19-32) M_{\odot}$ and that the bolometric luminosity is 4 to 6 times the Eddington luminosity, are robust as long as the slim disk interpretation of M82 X-1 energy spectrum appears valid.

\section{Discussion}

We have studied the M82 X-1 spectrum using archival XMM-Newton data of $105 \mathrm{ksec}$ exposure. Because of the high photon statistics, the continuum spectral model is tightly constrained compared with previous observations. The $p$-free disk model with $p=0.61$ gives a satisfactory fit, which is considered to be an evidence of the optically thick ADAF and presence of the slim disk in M82 X-1. We have applied the slim disk spectral model of Kawaguchi (2003), and estimated $M \approx(19-32) M_{\odot}$ and bolometric luminosity 4 to 6 times the Eddington luminosity.

Since the slim disk was theoretically proposed 18 years ago (Abramowicz et al. 1988), observational evidence of slim disks have been accumulating. For example, the brightest X-ray spectra of the black hole binary XTE J1550-564 are fitted with the $p$-free model with a small $p(\sim 0.5-0.7)$, while the standard disk model $(p=0.75)$ is successful when the luminosity is lower (Kubota \& Makishima 2004), Also, disk oscillation in GRS 1915+105 is considered to be a limit-cycle oscillation between the standard disk state and slim disk state (Honma et al. 1991a,b); and, in fact, the measured $p$-values of the upper and lower branches are $\sim 0.5$ and $\sim 0.75$, respectively (Yamaoka 2001). Not only Galactic black holes, but also soft X-ray energy spectra of some narrow-line Seyfert 1 galaxies, are successfully explained by a slim disk model (Kawaguchi 2003; Kawaguchi, Pierens, \& Hure 2004; Kawaguchi 2004). 
Since ULXs are, by definition, very luminous objects, it is rather straightforward to conclude that their accretion disks are slim, rather than the standard. While standard accretion disks around $\gtrsim 20 M_{\odot}$ black holes have characteristic temperatures $\lesssim 0.8 \mathrm{keV}$ (see Section 1), slim disks can explain the observed high disk temperatures $(\sim 2.8 \mathrm{keV})$. Here, we briefly review why a slim disk produces such a hard spectrum (see Kawaguchi 2003 for more detail). (1) As mass accretion rate increases, the innermost radius of the slim disk can be less than three times the Schwartschild radius even in the Schwartschild geometry (Watarai et al. 2000), thus the innermost disk temperature will be higher. (2) The ratio of the electron scattering opacity to the absorption opacity increases with mass accretion rates. Thus, photons generated deeper in the disk, where the temperature is higher, can escape from the disk surface more easily. Thus, the local spectral shape gets closer to the so-called modified blackbody, rather than a standard blackbody (e.g., Rybicki \& Lightman 1979). (3) Furthermore, because of the low rate of absorption, energy exchange between photons and electrons via inverse Compton scattering (disk Comptonization) is enhanced.

Based on our fitting of the slim disk model, we have found M82 X-1 is shining at 4 to 6 times the Eddington luminosity. Although a standard disk may not exceed the Eddington limit, such a moderate super-Eddington luminosity is naturally explained in the slim disk model (e.g., Abramowicz et al. 1988; Watarai et al. 2000). A recent two-dimensional radiation-hydrodynamic numerical simulation reports that a slim disk is formed under supercritical accretion flow, and the disk luminosity can exceed the Eddington luminosity by several factors (Ohsuga al. 2005).

We have estimated the black hole mass in M82 X-1 as $(19-32) M_{\odot}$. Although we have not seen such a rather heavy stellar-mass black hole in our Galaxy, such black holes are not prohibited by stellar evolution theory (e.g., Fryer 1999). Actually, detailed evolution models of stellar binaries show very small generation rate of intermediate-mass black holes (Madhusudhan et al. 2006). A universal luminosity function for X-ray binaries extends toward the highest luminosity $\sim 10^{40} \mathrm{erg} \mathrm{s}^{-1}$ without any break (Grimm, Gilfanov \& Sunyaev 2003), and ULXs presumably correspond to the highest luminosity X-ray binaries. This scenario agrees with the binary evolution synthesis model of ULXs by Rappaport, Podsiadlowski \& Pfahl (2005), which strongly suggests ULXs are stellar-mass black hole binaries.

In conclusion, the archetypal ULX M82 X-1 harbors a rather heavy but still stellarmass black hole shining at several times the Eddington luminosity. The slim disk model is reasonably successful in explaining the X-ray energy spectrum of M82 X-1. We do not find any evidence for an "intermediate-mass black hole" which may not be produced by ordinary stellar evolution. Since the high disk luminosity and temperature are not specific to M82 $\mathrm{X}-1$, but rather common properties of ULXs, we propose that most ULXs are probably 
stellar black holes shining at super-Eddington luminosities. Intermediate black holes are not necessary to explain the X-ray energy spectra of ULXs.

TO acknowledges support from NASA Grant NNG04GB78A. TK thanks the financial supports from the Japan Society for the Promotion of Science (JSPS) Postdoctoral Fellowships. This research has made use of public data and software obtained from the $X M M-N e w t o n$ Science Archive (XSA), provided by the European Sapce Agency (ESA), and the High Energy Astrophysics Science Archive Research Center (HEASARC), provided by NASA's Goddard Space Flight Center. The authors thank John P. Lehan for useful comments and assistance in correcting grammatical errors in the manuscript.

\section{REFERENCES}

Abramowicz, M. A., Czerny, B., Lasota, J. P., \& Szuskiewicz, E., 1988, ApJ, 332, 646

Begelman M.C. 1978, MNRAS, 184, 53

Ebisawa, K., Życki, P., Kubota, A., Mizuno, T. \& Watarai, K. 2003, ApJ, 597, 780

Fiorito, R., \& Titarchuk, L., 2004, ApJ, 614, L113

Fukue. J, 2000, PASJ, 52, 829

Fryer. C. L., 1999, ApJ, 522, 413

Grimm, H. J., Gilfanov, M., \& Sunyaev, R., 2003, MNRAS, 339, 793

Hirano, A., Kitamoto, S., Yamada, T., Mineshige, S. \& Fukue, J. 1995, ApJ, 446, 350

Kaaret, P., Prestwich, A. H., Zezas, A., Murray, S. S., Kim, D.-W., Kilgard, R. E., Schlegel, E. M., \& Ward, M. J., 2001, MNRAS, 321, L29

Kawaguchi T., 2003, ApJ, 593, 69

Kawaguchi T., Pierens A. \& Huré J.-M., 2004, A\&A, 415, 47

Kawaguchi T., 2004, Porg. Theor. Phys. Suppl., 155, 120

Kubota, A., \& Makishima, K., 2004, MNRAS, 353, 980

Kubota, A., Ebisawa, K., Makishima, K. \& Nakazawa, K. 2005, ApJ, 631, 1062 


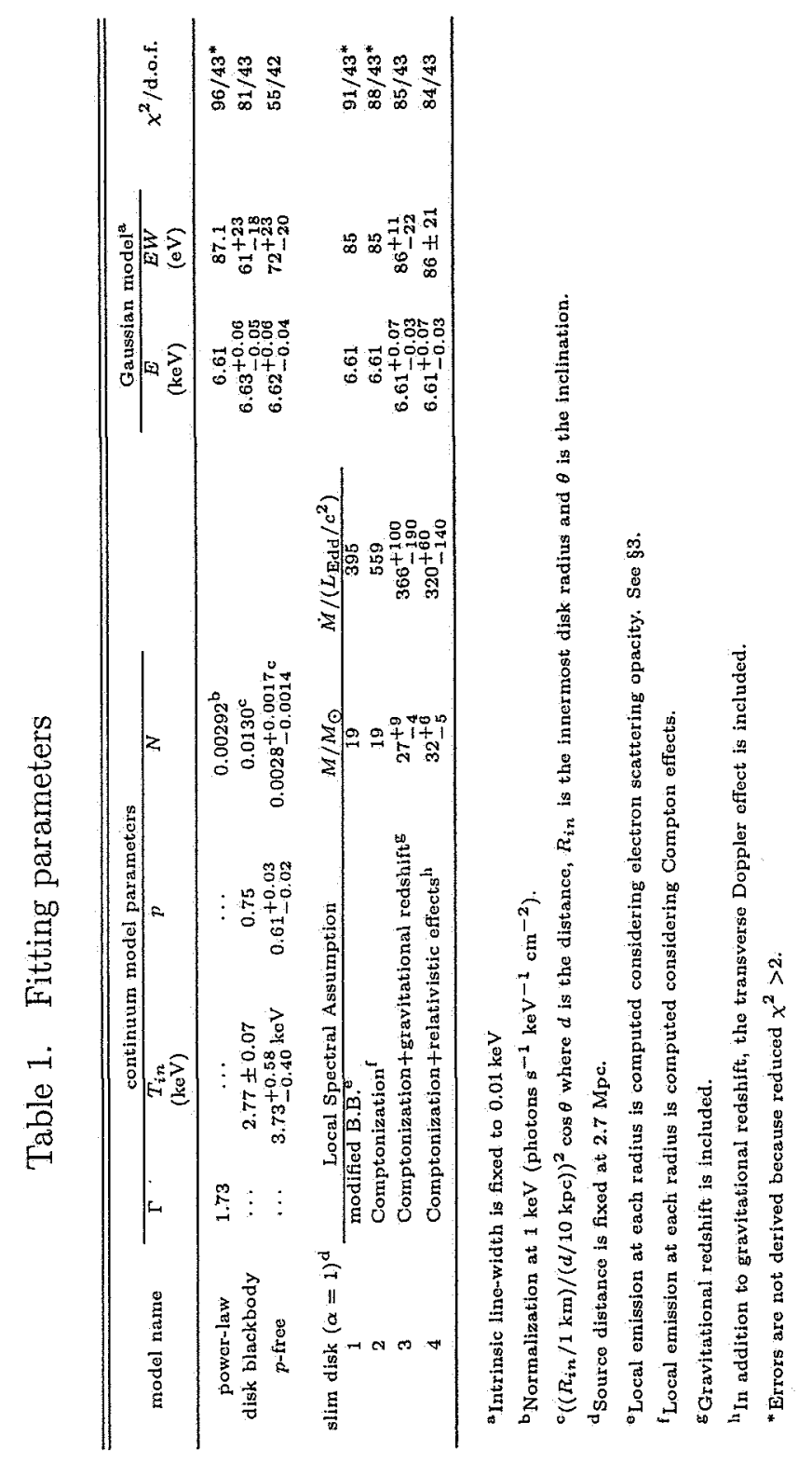


Honma F., Matsumoto R. \& Kato S., 1991, PASJ, 43, 147

Honma F., Matsumoto R., Kato S. \& Abramowicz M.A., 1991, PASJ, 43, 261

Madhusudhan N. et al. 2006, ApJ, 640, 918

Matsumoto, H., Tsuru, T. G., Koyama, K., Awaki, H., Canizares, C. R., Kawai, N., Matsushita, S., \& Kawabe, R., 2001, ApJ, 547, L25

Matsumoto, H., \& Tsuru, T. G. 1999, PASJ, 51, 321

Makishima, K. et al. 2000, ApJ, 535, 632

Mitsuda, K., Inoue, H., Koyama, K., Makishima, K., Matsuoka, M., Ogawara, Y., Suzuki, K., Tanaka, Y., Shibazaki, N., \& Hirano, T., 1984, PASJ, 36, 741

Miller, J. M., Fabian, A. C. \& Miller, M. C. 2004, ApJ, 607, 931

Miller, J. M., Fabbiano, G., Miller, M. C. \& Fabian, A. C. 2003, ApJ, 585, L37

Mineshige, S., Hirano, A., Kitamoto, S., Yamada, T., \& Fukue, J., 1994, ApJ, 426, 308

Mizuno, T., Kubota, A \& Makishima, K. 2001, ApJ, 554, 1281

Ohsuga, K. et al. 2005, ApJ, 628, 368

Okada, K. et al. 1998, PASJ, 50, 25

Ptak, A., \& Colbert, E. 2004, ApJ, 606, 291

Rieke, G. H. et al. 1980, ApJ, 238, 24

Rybicki, G. B., \& Lightman, A. P. 1979, Radiative Processes in Astrophysics, John Willey \& Sons, New York

Strohmayer, T. E., \& Mushotzky, R. F., 2003, ApJ, 586, L61

Shakura, N. I., \& Sunyaev, R. A. 1973, A\&A, 24, 337

Tsuru, T. G., Awaki, H., \& Koyama, K., 1997, PASJ, 49, 619

Rappaport, S. A., Padsiadlowski, Ph., \& Pfahl, E., 2005, MNRAS, 356, 401

Wang, Q. D., Yao, Y., Fukui, W., Zhang, S. N. \& Williams, R. 2004, ApJ, 609, 113

Watarai, K., Mizuno, T. \& Mineshige, S. 2001, ApJ, 549, L77 
Watarai, K., Fukue, J., Takeuchi, M. \& Mineshige, S. 2000, PASJ, 52, 133

Yamaoka, K. 2001, Ph.D. Thesis, University of Tokyo 


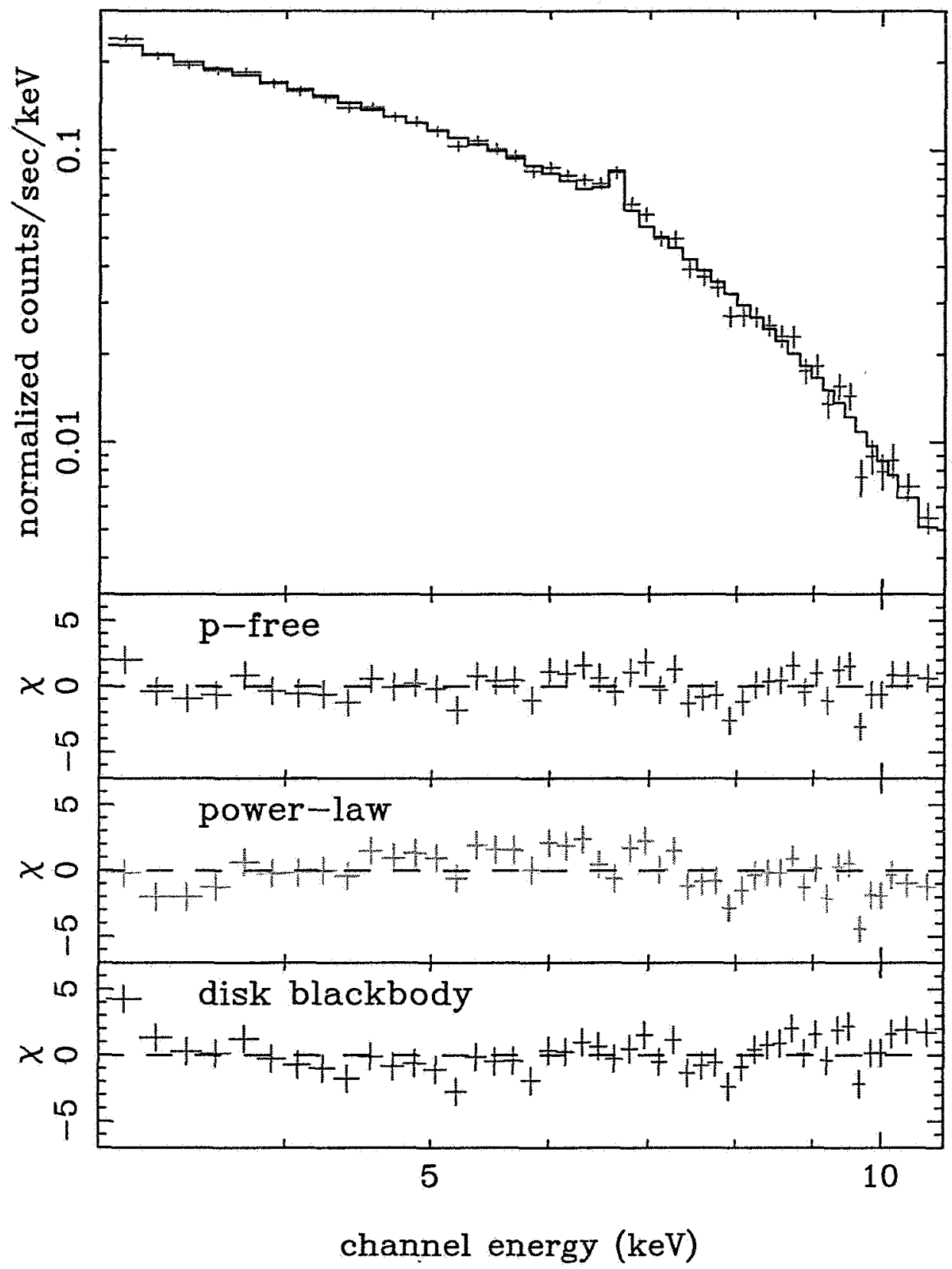

Fig. 1. - Folded spectrum of M82 X-1, fitted with the $p$-free disk blackboddy and narrow Gaussian model (top), and residuals for fitting with three different models, $p$-free, power-law, and disk blackboddy. 


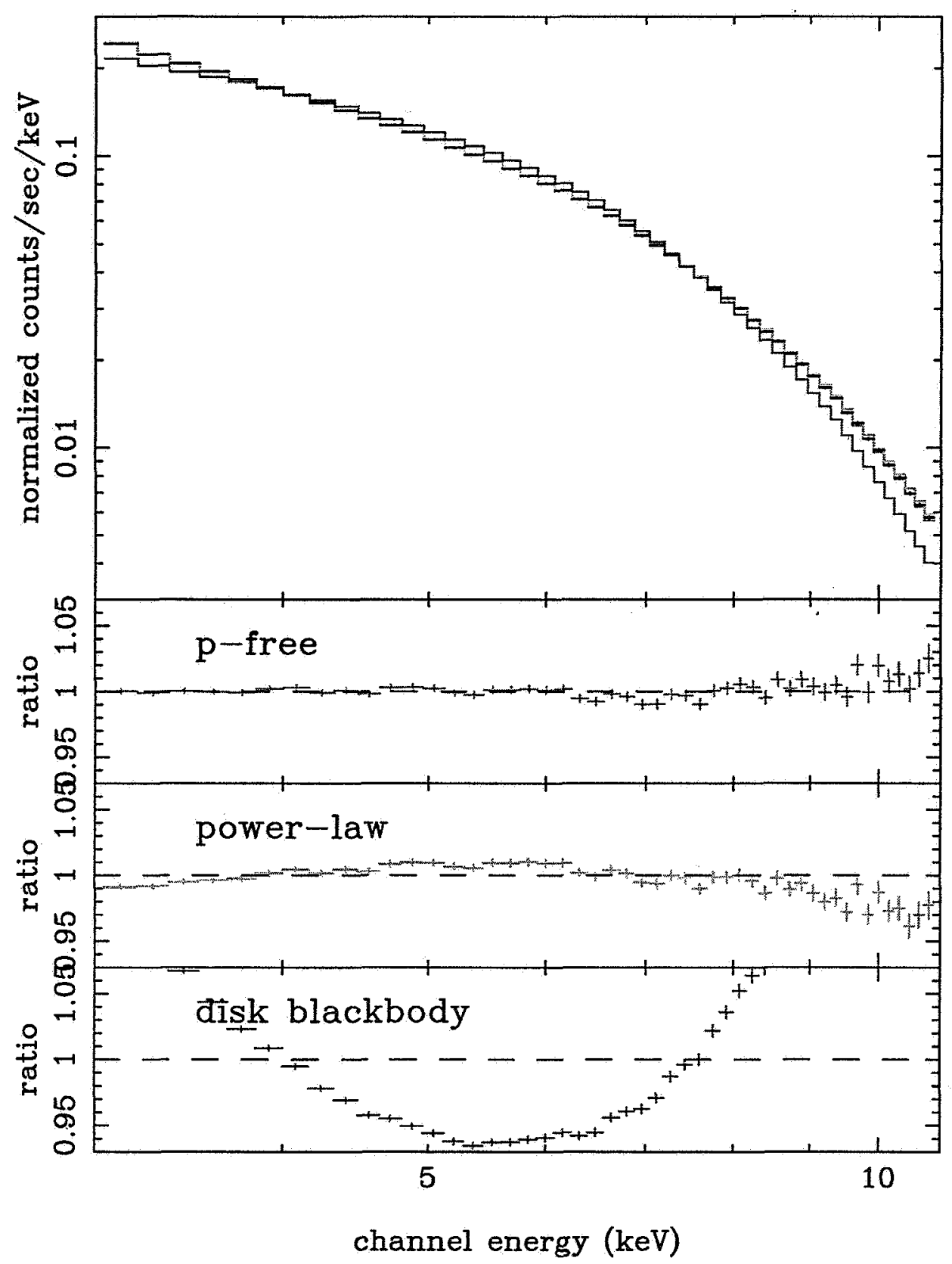

Fig. 2.- Comparison of the slim disk model by Kawaguchi (2003) and other models used to fit M82 X-1. A simulated spectrum was made from Kawaguchi's model 4 (see text) with $\alpha=1, M=30 M_{\odot}$ and $\dot{M}=350 L_{E d d} / c^{2}$ at $2.7 \mathrm{Mpc}$, and fitted with a power-law (index $=1.76)$, disk blackbody $\left(T_{\text {in }}=2.72\right)$, and $p$-free disk model $\left(p=0.54\right.$ and $\left.T_{\text {in }}=7.60\right)$. The top panel shows the simulated slim disk spectrum and the best-fit models, and the three bottom panels exibit the ratios of the simulated data to the $p$-free model, power-law and disk blackbody, respectively. Note that slim disk (solid line) and $p$-free (dashed line) models. are almost identical. 